\title{
Escherichia coli swimming is robust against variations in flagellar number
}

\author{
Patrick J Mears ${ }^{1,2}$, Santosh Koirala ${ }^{3}$, Chris V Rao ${ }^{3}$, Ido Golding ${ }^{1,2,4 *}$, \\ Yann R Chemla ${ }^{1,2 *}$
}

${ }^{1}$ Department of Physics, University of Illinois at Urbana-Champaign, Urbana, United States; ${ }^{2}$ Center for the Physics of Living Cells, University of Illinois at Urbana-Champaign, Urbana, United States; ${ }^{3}$ Department of Chemical and Biomolecular Engineering, University of Illinois at Urbana-Champaign, Urbana, United States; ${ }^{4}$ Verna and Mars McLean Department of Biochemistry and Molecular Biology, Baylor College of Medicine, Houston, United States

\begin{abstract}
Bacterial chemotaxis is a paradigm for how environmental signals modulate cellular behavior. Although the network underlying this process has been studied extensively, we do not yet have an end-to-end understanding of chemotaxis. Specifically, how the rotational states of a cell's flagella cooperatively determine whether the cell 'runs' or 'tumbles' remains poorly characterized. Here, we measure the swimming behavior of individual E. coli cells while simultaneously detecting the rotational states of each flagellum. We find that a simple mathematical expression relates the cell's run/tumble bias to the number and average rotational state of its flagella. However, due to inter-flagellar correlations, an 'effective number' of flagella—smaller than the actual number-enters into this relation. Data from a chemotaxis mutant and stochastic modeling suggest that fluctuations of the regulator CheY-P are the source of flagellar correlations. A consequence of inter-flagellar correlations is that run/tumble behavior is only weakly dependent on number of flagella.
\end{abstract} DOI: 10.7554/eLife.01916.001

*For correspondence: igolding@ illinois.edu (IG); ychemla@illinois. edu (YRC)

Competing interests: The author declares that no competing interests exist.

Funding: See page 17

Received: 19 November 2013 Accepted: 29 December 2013 Published: 11 February 2014

Reviewing editor: Michael Laub, Massachusetts Institute of Technology, United States

(c) Copyright Mears et al. This article is distributed under the terms of the Creative Commons Attribution License, which permits unrestricted use and redistribution provided that the original author and source are credited.

\section{Introduction}

Many species of bacteria swim by rotating helical filaments called flagella (Berg, 2004). A typical Escherichia coli cell is propelled by a bundle composed of multiple flagella. Each flagellum is controlled by a rotary motor that can switch between clockwise (CW) and counter-clockwise (CCW) rotation. When flagella on a cell rotate CCW, the cell swims along an approximately straight path called a 'run'. When some of the flagella rotate $\mathrm{CW}$, the bundle is disrupted causing an abrupt change in direction called a 'tumble' (Macnab and Ornston, 1977). E. coli modulates the probability of being in one of these two swimming states in response to its environment, allowing it to navigate chemical, temperature, and light gradients (Berg and Brown, 1972; Berg, 2004). At any point in time, the probability that a flagellar motor rotates $\mathrm{CW}$ is determined by the concentration of phosphorylated signaling protein $\mathrm{CheY}$ (CheY-P). Coupling CheY phosphorylation to chemicals from the environment allows the cell to bias its random walk and migrate towards more favorable conditions. This biased random walk is called chemotaxis, and serves as a model for understanding how living organisms process information (Berg and Brown, 1972; Wadhams and Armitage, 2004; Shimizu et al., 2010).

Tremendous progress has been made towards elucidating the mechanism of bacterial chemotaxis. The relationship between the chemotaxis signaling network and the CCW/CW rotational bias of the individual flagellar motor is now well mapped ([Block et al., 1982; Cluzel et al., 2000; Sourjik and Berg, 2002; Yuan et al., 2012]; for a review see Berg, 2004), and has also been described using detailed mathematical models (Emonet et al., 2005; Jiang et al., 2010; Shimizu et al., 2010). Despite this wealth of knowledge, how the CCW/CW states of individual motors collectively determine the 
eLife digest Escherichia coli is a rod-shaped bacterium commonly found in the lower intestines of humans and other warm-blooded animals. While most strains of E. coli are harmless, including most of those found in the human gut, some can cause diseases such as food poisoning. Due to its close association with humans and the fact that it is easy to grow and work with in the laboratory, $E$. coli has been intensively studied for over 60 years.

Many bacteria are capable of 'swimming' by using one or more flagella. These rotating whip-like structures are each driven by a reversible motor, and they act a bit like a propeller on a boat. While some bacteria have only a single flagellum, others, such as $E$. coli, have multiple flagella distributed over the cell surface. Rotating all their flagella in a counterclockwise direction allows the bacterium to swim - and it has been proposed that the clockwise movement of at least one flagellum will cause the bacterium cell to stop swimming and start tumbling.

E. coli is able to control the time it spends swimming or tumbling to move towards a nutrient, such as glucose, or away from certain harmful chemicals. However, the details of how the number of flagella and the direction of rotation (clockwise or counterclockwise) influence the motion of the bacterium are not fully understood.

Now, Mears et al. have used 'optical tweezers' to immobilize individual E. coli cells under a microscope, and then track both their swimming behavior and the movements of their flagella. This revealed that the individual flagella on the same cell tend to move in a coordinated way. Therefore, whilst tumbling could be caused by a single flagellum stopping swimming behavior, it often involved a concerted effort by many of the cell's flagella.

After observing that $E$. coli cells with more flagella spent less time tumbling than would be predicted if a single flagella always 'vetoed' swimming, Mears et al. propose a new mathematical relationship between the number of flagella on the cell, the direction of rotation, and the resulting probability that the cell will tumble. This work shows that swimming behavior in bacteria is less affected by variations in the number of flagella than previously thought-and this phenomenon may provide evolutionary advantages to $E$. coli. The next step is to explore the mechanism by which bacteria coordinate their flagella.

DOI: 10.7554/eLife.01916.002

run/tumble swimming behavior of the whole, multi-flagellated cell remains poorly understood. The number of flagella on an individual swimming cell can vary greatly, from one to more than ten (CohenBen-Lulu et al., 2008) (Figure 1-figure supplement 1), yet very few studies are available to indicate how flagellar number affects swimming behavior. The only direct measurements of flagellar dynamics in swimming cells have been limited to short durations $(\sim 1 \mathrm{~s})$ (Turner et al., 2000; Darnton et al。, 2007). The absence of long-term observations has precluded the development of a detailed mapping between flagellar state and cell swimming behavior. As a result, most theoretical models of bacterial chemotaxis are limited to treating an individual motor, or simply assume that all cells have a single flagellum (Bray et al., 2007; Kalinin et al., 2009; Matthaus et al., 2009; Jiang et al., 2010; Flores et al., 2012). Quantifying the mapping from single-flagellum state to whole-cell swimming behavior thus remains a missing link to developing an end-to-end picture of bacterial chemotaxis.

A number of theoretical models have been put forward in an attempt to describe this mapping. One such model invokes a 'voting' mechanism, in which cells tumble only if a majority of flagella rotate CW (Ishihara et al., 1983; Spiro et al., 1997; Andrews et al., 2006; Vladimirov et al., 2008; Jiang et al., 2010). However, by observing fluorescently labeled flagella during individual tumbles, Turner et al. established that CW rotation of a single flagellum is sufficient to 'veto' a run (Turner et alo, 2000) (Figure 1A). Refined versions of this 'veto model' were recently developed (Vladimirov et al., 2010; Sneddon et al., 2012), based on careful, slow-motion observations of tumbles (Darnton et al., 2007). However, the extent to which these details are relevant for modeling swimming behavior is unknown, because no measurements have directly correlated long-term swimming behavior with flagellar activity in the same cell.

In this study, we present simultaneous, prolonged observations of individual flagella and whole-cell swimming. Using an optical trap to hold a swimming cell (Min et al., 2009) while simultaneously imaging its fluorescently labeled flagella, we relate directly the number and state of each flagellum on a cell to its swimming behavior. Our measurements establish a simple mapping between CCW/CW flagellar 
A

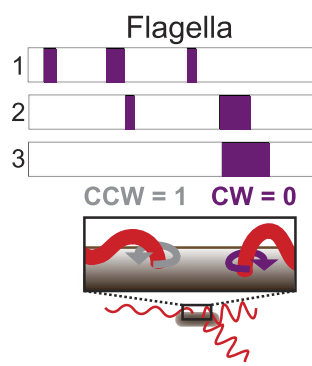

C

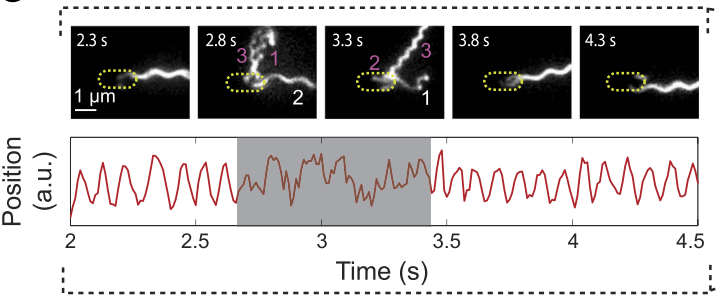

Cell

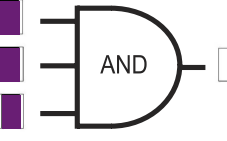

Run $=1 \quad$ Tumble $=0$

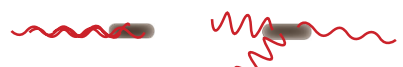

$\sqrt{2}$

D
B

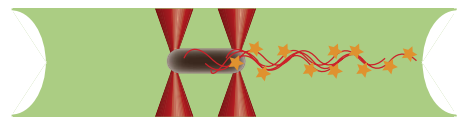

E

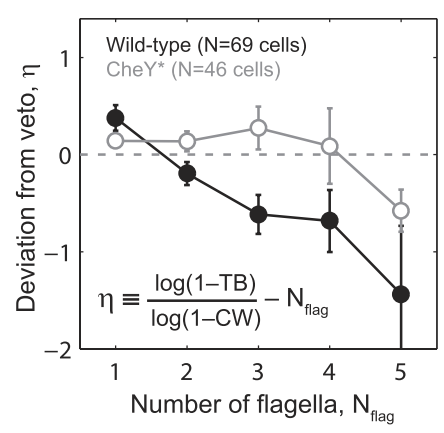

Figure 1. Wild-type E. coli cells deviate from the 'veto' model. (A) The mapping relating the run/tumble state of the cell to the CCW/CW state of its flagella according to the veto model. Schematic time trace from a cell with 3 flagella, showing CW (purple) and CCW (white) intervals for each flagellar motor and the resulting tumbles (black) and runs (white) of the cell. The veto model corresponds to an AND gate, by which cell runs only occur when all flagella rotate $\mathrm{CCW}$ (where $\mathrm{CCW}=1, \mathrm{CW}=0$, run $=1$, and tumble $=0$ ). (B) Schematic of a cell held by two optical traps (red cones) in the fluorescence excitation volume (green) within the sample chamber. (C) Representative data trace from a trapped cell with three flagella. Still images of fluorescently labeled flagella at 0.5 -s intervals (top). The approximate location of the unlabeled cell body is indicated by a dashed yellow line. Flagella rotating CW (purple) and CCW (white) are numbered in frames in which they appear distinct. Corresponding cell-body rotation signal for the same cell (red line, bottom) as detected from deflections of the trapping laser. Tumbles (shaded area) were determined from the erratic cell-body rotation signal. (D) Long-term time trace of CCW/CW flagellar rotation state and run/tumble cell swimming state. CW intervals (purple, top) for each flagellum were determined from the fluorescence images. Tumbles (black, bottom) were determined from the cell-body rotation signal. (E) Mean deviation $\eta$ from the veto model vs number of flagella per cell. Wild-type cells (solid black circles) with multiple flagella deviate significantly from the model ( $p=0.0003, N=69$ cells). CheY* cells (open gray circles; $N=46$ cells) match the model ( $p=0.77$ ). Error bars denote SEM. See 'Materials and methods' for more details.

DOI: 10.7554/eLife.01916.003

The following figure supplements are available for figure 1:

Figure supplement 1. Distribution of flagellar number.

DOI: 10.7554/eLife.01916.004

Figure supplement 2. Instrument layout.

DOI: 10.7554/eLife.01916.005

Figure supplement 3. Laser temporal interlacing scheme.

DOI: 10.7554/eLife.01916.006

Figure supplement 4. Sample data from representative cells.

DOI: 10.7554/eLife.01916.007

Figure supplement 5. Cells with flagella in the curly-1 state rarely run.

DOI: 10.7554/eLife.01916.008 
rotation and run/tumble bacterial swimming state for cells with arbitrary numbers of flagella. Surprisingly, we find that $E$. coli cells wild-type for chemotaxis do not strictly comply with the veto model, because the states of individual flagella in the same cell are strongly coupled. Flagella do not switch independently, and as a result, tumbles typically involve many of the flagella on the cell. The behavior of multi-flagellated cells can still be mapped to the simple veto model by renormalizing the flagellar number to a lower effective number of independent flagella. As for the cause of inter-flagella coupling, our data strongly favor a mechanism involving fluctuations of the signaling network rather than direct, physical interactions between flagella. This model is supported by the observation that mutant cells in which flagellar switching is decoupled from the chemotaxis network do obey the simple veto model, as well as by stochastic simulations of the swimming behavior.

\section{Results}

\section{The tumble bias of wild-type cells deviates from the predictions of the veto model}

According to the veto model, a cell tumbles whenever any one flagellum rotates CW (Figure 1A). Thus, the probability that a cell runs equals the probability that all of its flagella remain CCW. As a consequence, cells with more flagella are expected to tumble more, since there is a higher chance that at least one flagellum will deviate from the consensus and 'veto' the run. These predictions can be stated mathematically, under the assumption that the rotational direction of each flagellum is independent of the other flagella. In a cell with $N_{\text {flag }}$ flagella, the average tumble bias TB-the fraction of time a cell spends tumbling-will be given by

$$
T B=1-(1-C B)^{N_{\text {flag }}}
$$

where CB is the average clockwise bias-the fraction of time the cell's flagella rotate CW ('Materials and methods').

To test this prediction, we quantified the swimming behavior of individual E. coli cells wild-type for chemotaxis (strain HCB1660 [Turner et al., 2010]; see 'Materials and methods', Table 1) using an instrument combining optical tweezers and epi-fluorescence imaging (Figure 1B and Figure 1-figure supplement 2). The instrument allowed us to measure simultaneously run/tumble behavior and flagellar dynamics in the same cell. The optical trap was used to hold each end of a single cell in place and the light scattered by the cell was utilized to monitor its swimming behavior, as described previously (Min et al., 2009, 2012). As shown in Figure 1C (Video 1), cell runs were identified from oscillatory time signals due to cell body rotation at a frequency of $\sim 10 \mathrm{~Hz}$. Cell tumbles were identified as periods of erratic motion during which the flagellar bundle was disrupted (Min et al., 2009). Flagella were fluorescently labeled using the method of Turner et al. (2010). High speed, epi-fluorescent, stroboscopic imaging (Turner et alı, 2000; Figure 1-figure supplement 3) was used to resolve individual flagella (Figure 1C). Since the trapped cell remained in the field of view for a prolonged period, flagella were observed through multiple runs and tumbles (typically $\sim 5$ events), limited by the time until flagella became too dim to discern due to photobleaching ( 8 to $40 \mathrm{~s}$ ). The rotational direction of each flagellum was determined by observing its shape during 100-ms time windows. As shown by Darnton et al. (2007), flagella may take on different helical waveforms depending on their rotational state. These waveforms, termed 'normal', 'semi-coiled', 'curly-1', and 'curly-2', can be visually identified based on their pitch and wavelength (Figure 1C, Figure 1-figure supplement 4). CCW rotating flagella were identified based on the normal conformation, which they have been shown to adopt exclusively (Darnton et al., 2007), while CW rotating flagella were identified by their curly-1 or semi-coiled shape. From the identification of CCW and CW intervals, the cell's mean CW bias was determined by averaging the fraction of time that all the flagella on the cell spent CW ('Materials and methods').

Our assay allowed us to determine all the parameters in Equation (1) directly. For each cell, we measured the tumble bias (using the optical trap), flagellar number $N_{\text {flag, }}$ and $\mathrm{CW}$ bias (using fluorescence imaging). We used these values to compare our experimental data to the prediction of the veto model. Reorganizing Equation (1), we define the parameter $\eta$ :

$$
\eta \equiv \frac{\log (1-T B)}{\log (1-C B)}-N_{f l a g}
$$


Table 1. Strains and plasmids used in this work

\begin{tabular}{|c|c|c|c|}
\hline Strain & Genotype & Comments & Source \\
\hline HCB1660 & fliC::Tn5 $\left(\operatorname{Kan}^{\mathrm{R}}\right)$ & $\begin{array}{l}\text { 'wild type' Contains plasmid } \\
\text { pBAD33-flic } 219 \mathrm{C}\end{array}$ & $\begin{array}{l}\text { (Turner et al., 2010) } \\
\text { Gift of H Berg }\end{array}$ \\
\hline PM87 & cheBYZ::FRT, fliC::Tn5 (Kan) & $\begin{array}{l}\text { 'CheY*' Contains plasmids } \\
\text { pMS164 and pPM5 }\end{array}$ & This study \\
\hline RP437 & & Wild-type for chemotaxis & $\begin{array}{l}\text { (Parkinson and } \\
\text { Houts, 1982) }\end{array}$ \\
\hline SK109 & cheBYZ::Cm & & This study \\
\hline SK110 & cheBYZ::FRT & & This study \\
\hline SK112 & cheBYZ::FRT, fliC::Tn5 (Kan) & & This study \\
\hline \multicolumn{4}{|l|}{ Plasmids } \\
\hline pBAD33 flic ${ }^{\mathrm{s} 219 \mathrm{C}}$ & $\begin{array}{l}f_{i C^{S 219 C}} \text { under } P_{\text {araBAD }} \\
\text { promoter, } \mathrm{Cm}^{\mathrm{R}}, \mathrm{p} 15 \text { a origin }\end{array}$ & $\begin{array}{l}\text { Expresses mutant version of } \\
\text { FliC for fluorescent labeling }\end{array}$ & $\begin{array}{l}\text { (Turner et al., 2010) } \\
\text { Gift of H Berg }\end{array}$ \\
\hline pPM5 & $\begin{array}{l}\text { fli } C^{S 219 C} \text { under } P_{\text {araBAD }} \\
\text { promoter, Amp }{ }^{R} \text {, colE1 origin }\end{array}$ & $\begin{array}{l}\text { Expresses mutant version of } \\
\text { FliC for fluorescent labeling }\end{array}$ & This study \\
\hline pMS164 & $\begin{array}{l}\text { cheY }{ }^{D 13 K} \text { under } \mathrm{P}_{\text {lacop }} \\
\text { promoter, } \mathrm{Cm}^{\mathrm{R}}, \mathrm{pSC} 101 \text { origin }\end{array}$ & $\begin{array}{l}\text { Expresses constitutively } \\
\text { active version of CheY }\end{array}$ & $\begin{array}{l}\text { (Alon et al., 1998) } \\
\text { Gift of P Cluzel }\end{array}$ \\
\hline pDK46 & & Helper plasmid & $\begin{array}{l}\text { (Datsenko and } \\
\text { Wanner, 2000) }\end{array}$ \\
\hline pKD3 & & Template for $\mathrm{Cm}^{\mathrm{R}}$ cassette & $\begin{array}{l}\text { (Datsenko and } \\
\text { Wanner, 2000) }\end{array}$ \\
\hline pCP20 & & Helper plasmid & $\begin{array}{l}\text { (Cherepanov and } \\
\text { Wackernagel, 1995) }\end{array}$ \\
\hline
\end{tabular}

DOI: 10.7554/eLife.01916.009

which quantifies the deviation of the data from the veto model. Comparing Equation (2) to Equation (1), $\eta$ may also be interpreted as the difference between two terms: the number of flagella estimated from the veto model based on the cell's swimming behavior, and the number of flagella on the cell as determined by counting directly. Figure 1E (black circles) shows $\eta$ against the flagellar number $N_{\text {flag. }} \eta$ was calculated for each individual cell and then averaged over all cells with a given number of flagella. Unexpectedly, we found that wild-type cells with multiple flagella systematically deviated from the predicted behavior. Specifically, $\eta$ was consistently negative for cells with $N_{\text {flag }}>1$ (35/48 cells), indicating that cells with multiple flagella tumbled less than expected from the model. In the context of the veto model, the cells behaved as if they had a smaller number of flagella than what they actually had.

We first considered the possibility that a more detailed version of the veto model might explain the observed behavior and reconcile this discrepancy. A recent study by Sneddon et al. (2012) used the observations of Darnton et al. (2007) to refine the veto model. Specifically, the Sneddon model states that a cell with a minimum of $X \mathrm{CCW}$ flagella will run rather than tumble, provided the remaining $\left(N_{\text {flag }}-X\right) \mathrm{CW}$ flagella are in the curly-1 conformation only ( $X$ is a parameter in the model, with possible values in the range [1, $\left.\left.N_{\text {flag }}-1\right]\right)$. Thus, the simple veto model considered above corresponds to $X=N_{\text {flag, }}$ and the least perturbative refinement to the model corresponds to $X=N_{\text {flag }}-1$, in which a cell with a single curly-1 flagellum still runs. However, in our measurements we observed that cells with a single CW flagellum in the curly-1 state still tumbled $82 \%$ of the time (44 s of cumulative time in which one flagellum was in the curly-1 state; Figure 1-figure supplement 5). Modifying the Sneddon model to allow runs $18 \%$ of the time was not sufficient to reproduce the trend observed in Figure 1D, Figure 1figure supplement 5 .

\section{Wild-type tumbles typically involve multiple flagella}

To investigate the discrepancy between our data and the veto model, we next examined individual tumble events in greater detail. In agreement with the original observation of Turner et al. (2000), we found that $\mathrm{CW}$ rotation of a single flagellum was indeed sufficient to cause a tumble in multi-flagellated cells (Figure 1-figure supplement 4, samples A, D and E). However, we also observed that more 


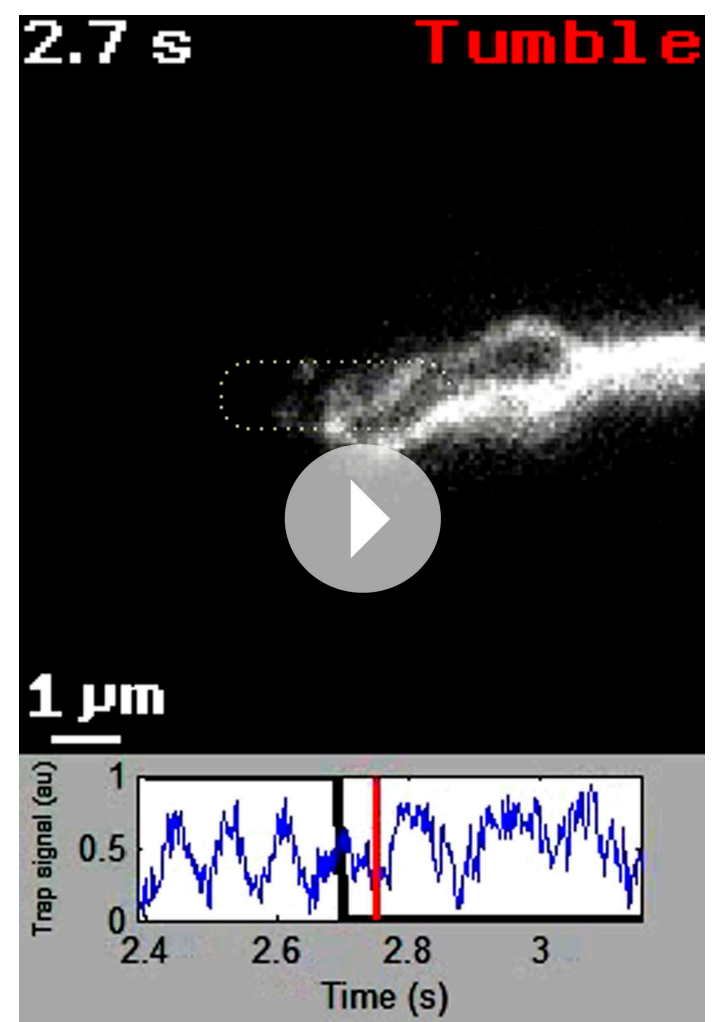

Video 1. Video of trapped wild-type cell with three labeled flagella as it runs and tumbles. Slow motion video of the wild-type cell in Figure $1 \mathrm{C}$ with three long, fluorescently labeled flagella. The approximate location of the unlabeled cell body is indicated by the dotted line. The trap signal used to determine runs and tumbles (bottom, scrolling blue curve) measures the position of the cell body in the trap as it rotates. At the beginning of the video (time stamp $=2.4 \mathrm{~s}$ ), all three flagella are in a bundle and the cell is running. One by one, the flagella switch to CW rotation (2.7-3.0 s), which disrupts the bundle and causes the cell to tumble. Flagella can be observed in all three waveforms, normal, semi-coiled, and curly-1. Near the end of the video, the flagella all return to CCW rotation and coalesce into a bundle, causing the cell to resume running (3.4 s). In addition to the three long flagella, a short flagellar stub is visible. The stub does not affect the swimming behavior and is not analyzed. Scale bar in bottom left corner is $1 \mu \mathrm{m}$. Frames were recorded at 400 frames per second, video shows every other frame at 20 frames per second.

DOI: 10.7554/eLife.01916.010 compare black and gray data points). Comparing 54 wild-type and $24 \mathrm{CheY}^{\star}$ cells with the same mean $\mathrm{CW}$ biases $(0.11 \pm 0.07$ vs $0.11 \pm 0.07$, mean \pm SD) we found that, on average, fewer CW rotating flagella participated in tumbles in the $\mathrm{CheY}^{*}$ strain (Figure 2C, open circles). Moreover, the number of participating flagella in the CheY* strain closely matched the expectation for cells with independently switching flagella (Figure 2C, dashed line). (This number deviates from unity and trends upwards with number of flagella simply because of the finite probability that two tumbles overlap by chance.) Our results indicate that when the signal for flagellar motors to switch their rotational state is decoupled from the chemotaxis network, the motors switch independently. Based on our interpretation of the wild-type data, we thus expect CheY* cells to

than half of tumbles in multi-flagellated cells $(56 \%$, $117 / 210$ events) actually involved multiple CW flagella. Figure 2A shows a representative trace from a wild-type cell with three flagella. There are times during each tumble in the trace where all three flagella are in a CW state. As shown in Figure 2C, the number of CW flagella 'participating' in a tumble (black circles) was significantly larger than would be expected if flagella were independently switching (gray dashed line, obtained from simulations of a cell with independent flagella; see 'Materials and methods'). Our results thus suggest that while a single CW flagellum is sufficient to induce a tumble (in agreement with a simple veto model), flagella are also coupled and may thus switch in groups, in a correlated fashion. Further evidence for interflagella coupling was obtained by calculating the cross-correlation between pairs of flagella on a given cell. We found a significant correlation between the rotational directions of pairs of flagella on the same cell (Figure 2D, black data points). This correlation persisted for $\sim 1 \mathrm{~s}$, the average duration of a tumble. Our findings are consistent with previous observations by Terasawa et al. on surface-immobilized cells (Terasawa et al., 2011). There, correlations between individual motors on the same cell were detected by monitoring beads attached to flagellar stubs, as opposed to complete flagella on swimming cells in our present work.

The source of inter-flagellar correlation remains under debate. Terasawa et al. observed that mutant cells, in which the concentration of CheY-P was decoupled from the chemotaxis network, displayed no correlations (Terasawa et al., 2011). This led us to likewise investigate the behavior of a strain, PM87, expressing a constitutively-active CheY (CheY ${ }^{\mathrm{D} 13 \mathrm{~K}}$ [Alon et al., 1998], denoted CheY*, see Table 1 and 2). The protein was exogenously expressed, with the expression level chosen such that the population-averaged tumble bias matched that of wild-type cells ('Materials and methods'). A representative trace from a $\mathrm{CheY}^{*}$ cell with three flagella is shown in Figure 2B (see also Figure 1-figure supplement 4, and Video 2). Upon inspection, flagellar switching appears far less correlated than in wild-type cells (Figure 2D,

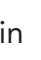

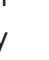

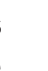



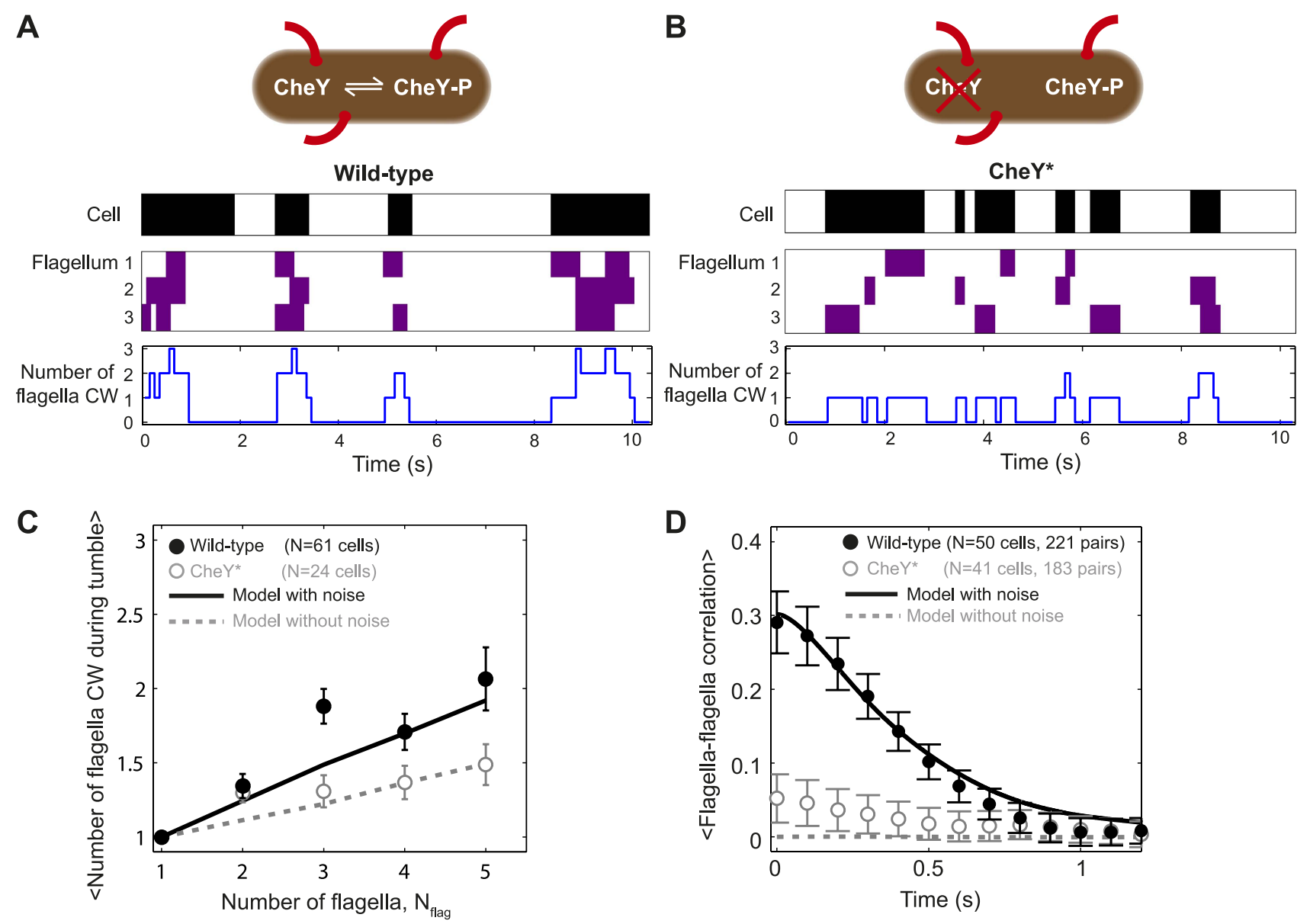

Figure 2. Tumbles in wild-type cells involve multiple CW flagella. (A) A typical time trace from a wild-type cell with 3 flagella. Colors indicate runs/ tumbles (white/black, top) and CCW/CW (white/purple, middle). The blue line (bottom) shows the corresponding number of CW flagella at each time point. (B) Same as (A) for a typical CheY* cell with 3 flagella. (C) Mean of the maximum number of CW flagella during a tumble vs number of flagella per cell. Consistently more flagella are $\mathrm{CW}$ during tumbles in the wild-type (black circles; $N=61$ cells) compared to the Che $Y^{\star}$ strain (open gray circles; $N=24$ cells). Simulations incorporating fluctuations in CheY-P (black line) and without fluctuations (gray dashed lines) reproduce the observed trends (simulations detailed in the text and Figure 4). (D) Cross-correlation between flagella pairs, averaged over all pairs and all cells. Wild-type (black circles) match simulations with fluctuations in CheY-P (black line). CheY* strain (open gray circles) matches simulations without fluctuations in CheY-P (gray dashed line), which exhibit almost no correlation. Error bars denote SEM. See 'Materials and methods' for more details.

DOI: 10.7554/eLife.01916.011

The following figure supplements are available for figure 2:

Figure supplement 1. Flagellar transition rates vs number of flagella per cell.

DOI: 10.7554 /eLife.01916.012

Figure supplement 2. Flagellar transition rates vs number of flagella per cell.

DOI: 10.7554/eLife.01916.013

adhere to the simple veto model. As shown in the plot of $\eta$ in Figure 1D (open circles), CheY* cells indeed match the veto model closely $(\eta=-0.08 \pm 0.15$, mean \pm SEM). The existence of correlations between flagella states in wild-type cells may thus explain why cells with multiple flagella deviate from the veto model.

\section{Wild-type behavior can be described by a veto model with a lower effective number of flagella}

Our results so far suggest that, while wild-type cells obey the fundamental premise of the veto modelthat is, a single CW flagellum is sufficient to induce a tumble-the presence of inter-flagella correlations leads to the failure of Equation (1) in relating the observed CW bias and tumble bias. To describe the 
Table 2. Primers used in this work

\begin{tabular}{lll} 
Primer & Sequence & Comments \\
\hline SK140F & $\begin{array}{l}\text { TGCGTGGTCAGACGGTGTATGCGCTAAGTAAGGATTAACG } \\
\text { GTGTAGGCTGGAGCTGCTTC }\end{array}$ & cheBYZ deletion forward \\
\hline SK140R & $\begin{array}{l}\text { GCCTGATATGACGTGGTCACGCCACATCAGGCAATACAAA } \\
\text { CATATGAATATCCTCCTTAG }\end{array}$ & cheBYZ deletion reverse \\
\hline SK141F & CCTTAAACCCGACGGATTGC & cheBYZ deletion check forward \\
\hline SK141R & TTGCTGCCACACATCAAGC & cheBYZ deletion check reverse \\
\hline SK163F & AGGGTTATTGTCTCATGAGC & pZE11 sequencing forward \\
\hline SK163R & GTTTTATTTGATGCCTCTAG & PZE11 sequencing reverse \\
\hline PM7F & GGG GACGTC ATCGATGCATAATGTGCCTG & amplify ParaA fliC $P_{\text {S219C forward }}$ \\
\hline PM7R & GGG GTCGAC TTAACCCTGCAGC & amplify ParabA fliC 5219 reverse
\end{tabular}

DOI: 10.7554/eLife.01916.014

relation between single flagellar state and whole-cell behavior successfully, this expression must then be modified to account for flagellar correlations. To this end, we examined the relation between $\mathrm{CW}$ bias and tumble bias in all individual cells having a given flagellar number (Figure 3A,B). Equation (1) defines a single curve, along which the $\mathrm{CW}$ bias and tumble bias of all cells with $N_{\text {flag }}$ flagella should lie (dashed line in Figure 3A,B). As expected, the $\mathrm{CheY}^{\star}$ cells follow these predicted curves closely for all $N_{\text {flag }}$ values (Figure $\left.3 B\right)\left(R^{2}=0.89\right)$. In contrast, wild-type cells with multiple flagella consistently fall below the predicted curves (Figure 3A) (35/48 cells).

Based on our observation that wild-type cells exhibited $\eta$ values consistent with cells with a lower number of flagella than the actual value, we hypothesized that wild-type behavior may be described within the framework of the veto model by allowing the parameter $N_{\text {flag }}$ in Equation (1) to deviate from the actual flagellar number. As shown in Figure 3A (solid lines), using the flagellar number as a fitting parameter (now denoted $N_{\text {eff }}$ ) indeed allows for a good match for the wild-type data $\left(R^{2}=0.85\right)$. In this revised equation, $N_{\text {eff }}$ can be thought of as the 'effective' number of independent flagella on a cell, which captures the fact that flagella in wild-type cells switch in a correlated manner. Consistent with this picture, the effective number of flagella $N_{\text {eff }}$ is consistently smaller than the actual flagellar number $N_{\text {flag }}$ (Figure $3 C$, black circles; $N_{\text {eff }}$ can be approximated by $N_{\text {eff }}=1.27 \times N_{\text {flag }}{ }^{0.5}$, see Figure 3-figure supplement 1). As a control, estimating $N_{\text {eff }}$ for $\mathrm{CheY}^{\star}$ cells produces values very close to the original flagellar number $N_{\text {flag }}$ (Figure 3B, solid line and Figure 3C, open circles). The introduction of the parameter $N_{\text {eff }}$ allows us to formulate a generalized veto model, which describes the mapping between the $\mathrm{CW}$ bias and tumble bias for both wild-type and $\mathrm{CheY}^{\star}$ cells. The generalized model now defines a universal curve,

$$
1-T B=(1-C B)^{N_{\text {eff }}}
$$

along which all individual cells of both genotypes should lie (using $N_{\text {eff }}=N_{\text {flag }}$ for CheY* strain and the best fit value of $N_{\text {eff }}$ for wild-type). As seen in Figure 3D, this expression successfully collapses all single-cell data from both strains and all flagellar numbers.

\section{A theoretical model incorporating CheY-P fluctuations reproduces wild-type swimming behavior}

Our results show that $E$. coli cells adhere to the veto model, but that inter-flagellar correlations lead to a renormalization of the effect of flagellar number. What is the source of these flagellar correlations? The absence of correlations in the $\mathrm{CheY}^{\star}$ strain and its adherence to a simple veto model provide an important clue to understanding the mechanism of inter-flagellar coupling. In wild-type cells, CheY-P levels are subject to phosphorylation and de-phosphorylation reactions by chemotaxis network components and are believed to fluctuate in time (Korobkova et al., 2006; Sneddon et al., 2012). In contrast, in the mutant strain, $\mathrm{CheY}^{*}$ levels are decoupled from the network and are thus expected to be constant over the timescales of interest (Korobkova et al., 2006; Min et al., 2009). Terasawa et al. proposed that fluctuations in CheY-P levels may thus provide a mechanism by which the CW biases of multiple flagella on a cell can be coupled (Terasawa et al., 2011). To test whether such a mechanism could account for the different features of bacterial swimming observed in our study, we performed simulations of whole-cell swimming driven by the chemotaxis network. In particular, we investigated 


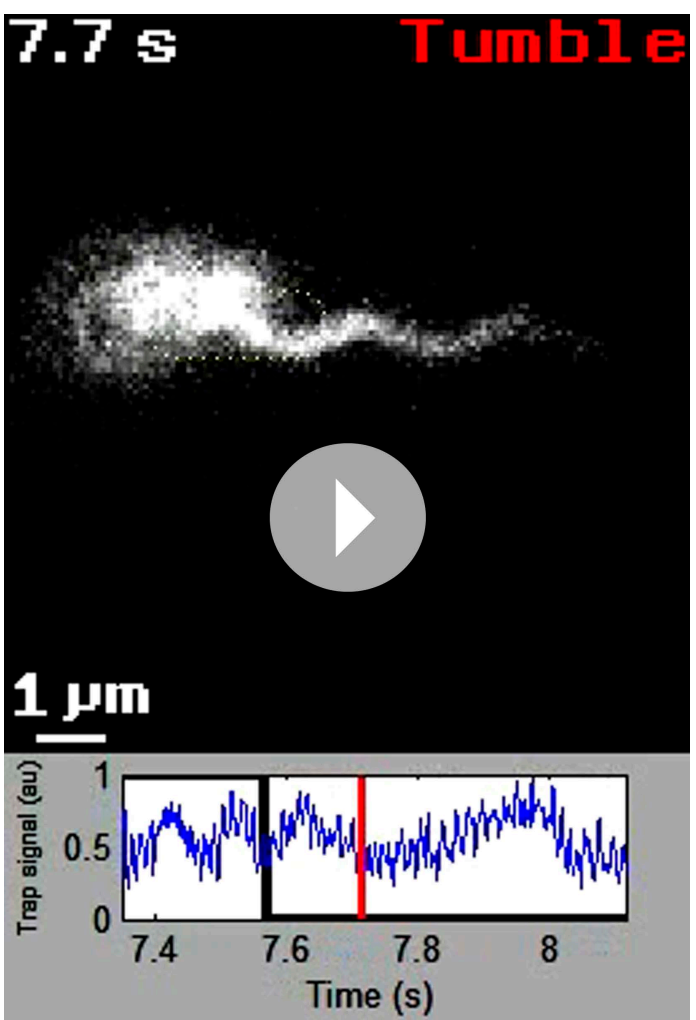

Video 2. Video of trapped $\mathrm{CheY}^{*}$ cell with two labeled flagella as it runs and tumbles. Slow motion video, similar to Video 1, of a trapped $\mathrm{CheY}^{*}$ cell with two fluorescently labeled flagella. Still images from this cell are shown in Figure 1-figure supplement 4, sample D. The approximate location of the unlabeled cell body is indicated by the dotted line. At the beginning of the video (time stamp $=7.4 \mathrm{~s}$ ), both flagella are in a bundle, rotating $\mathrm{CCW}$ in the normal waveform, and the cell is running. At $7.6 \mathrm{~s}$, one flagellum switches to $\mathrm{CW}$ rotation and transitions to the semi-coiled waveform, which disrupts the bundle and causes the cell to tumble. At the $8.5 \mathrm{~s}$, the semi-coiled flagellum returns to CCW rotation and both flagella coalesce into a bundle, causing the cell to resume running. Scale bar in bottom left corner is $1 \mu \mathrm{m}$. Frames were recorded at 100 frames per second, video plays at 20 frames per second. DOI: 10.7554/eLife.01916.015 bution of possible sequences of flagellar waveforms during tumbles. In particular, the sequence of states from normal to semi-coiled to curly-1 that we observed was described by Darnton et al. (2007) as a 'canonical tumble'. Although we cannot rule out that runs and tumbles in the optical trap are different in some ways than those in free swimming cells (Min et al., 2009), our results are in qualitative agreement with these previous observations.

Our measurements also reveal the relationship between the cell's run/tumble state and the CCW/ $\mathrm{CW}$ rotational state of its flagella. In a multi-flagellated wild-type cell, a single CW flagellum (either semi-coiled or curly-1) is sufficient to induce a tumble, in agreement with the simple veto model (Turner et al., 2000; Darnton et al., 2007). However, the number of CW flagella during a tumble typically exceeds that expected from a cell with independently switching flagella (Figure 2C). The high fraction of CW flagella during tumbles in wild-type cells is in qualitative agreement with previous measurements by Turner et al. (2000), who observed that a majority of tumbles involved multiple flagella leaving the bundle. Our measurements using the $\mathrm{CheY}^{\star}$ strain provide an important piece of evidence 


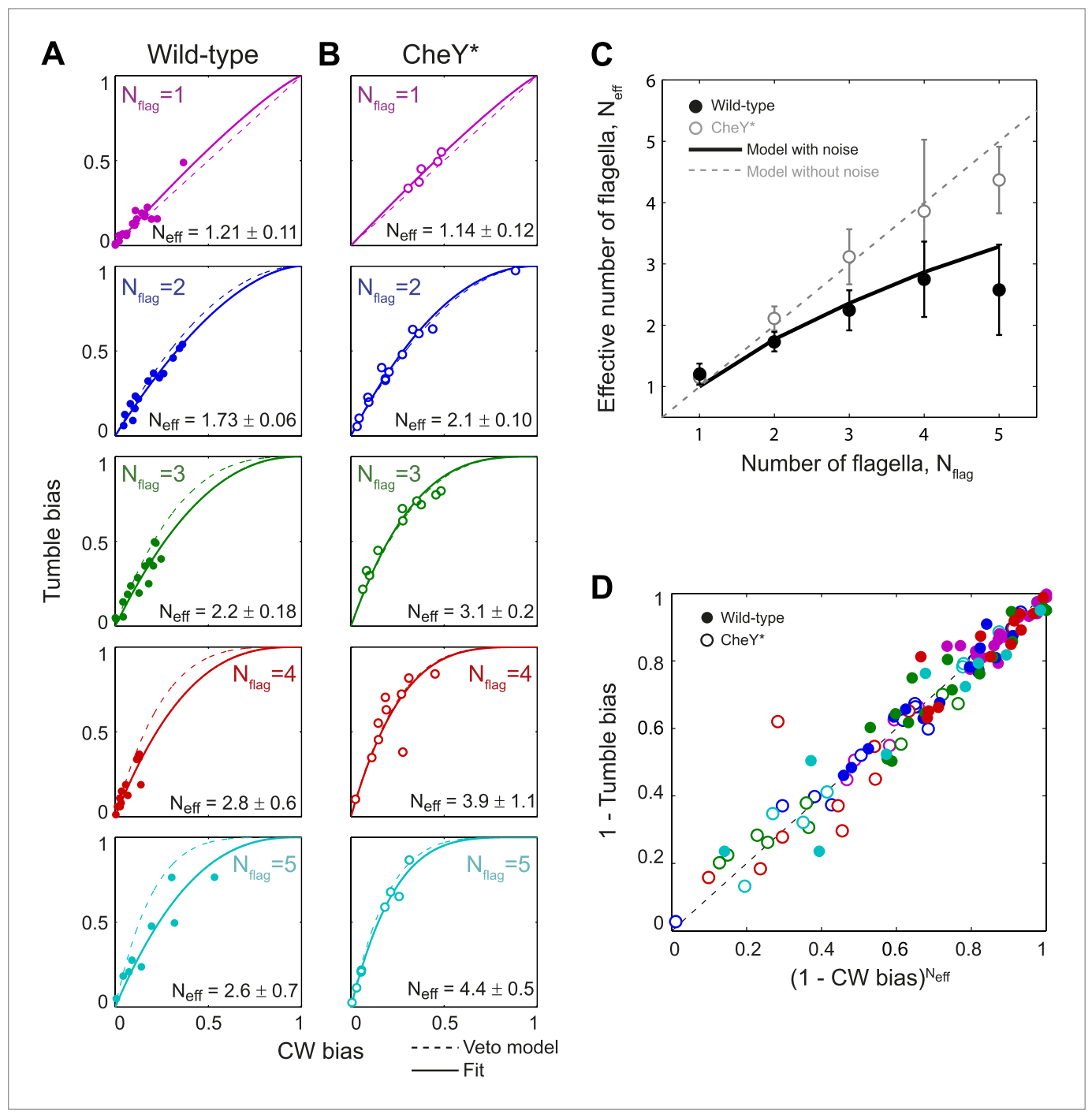

Figure 3. Wild-type behavior matches the veto model for cells with a lower effective number of flagella. (A) Tumble bias vs CW bias for individual wild-type cells $(N=69)$, plotted separately for different numbers of flagella per cell $\left(N_{\text {flag }}=1\right.$, purple; 2 , blue; 3 , green; 4 , red; 5 , cyan). The prediction from the veto model in Equation (1) (dashed lines) does not match the data for cells with multiple flagella $\left(R^{2}=0.88,0.60,0.41,0.39\right.$ for $\left.N_{\text {flag }}=2,3,4,5\right)$. The data were fit (solid lines) to Equation (1), while allowing the number of flagella to be used as a fitting parameter, $N_{\text {eff. }}$ Error bars denote SD. (B) Same as (A) for CheY* (open circles, same color code as $[\mathbf{A}] \mathrm{N}=46$ cells). The veto model prediction (dashed lines) matches the data well $\left(R^{2}=0.91,0.97,0.93,0.67,0.98\right.$ for $\left.N_{\text {flag }}=1,2,3,4,5\right)$. Fits (solid lines) yield $N_{\text {eff }}$ values almost identical to $N_{\text {flag. }}$. (C) Fitted $N_{\text {eff }}$ values vs number of flagella per cell for wild-type (black circles) and $\mathrm{CheY}^{\star}$ (open gray circles) cells. Simulations (described in the text) reproduce the observed trends. (D) Data points from individual wild-type (solid circles) and $\mathrm{CheY}^{*}$ (open circles) cells all collapse onto a single line when using $N_{\text {eff }}$ from fits to wild-type data in (A) and the actual flagellar number $N_{\text {flag }}$ for $C h e Y^{\star}$ cells in (B). Error bars denote SEM. See 'Materials and methods' for more details.

DOI: 10.7554/eLife.01916.016

The following figure supplements are available for figure 3:

Figure supplement 1. Fit to $\mathrm{N}_{\text {eff }} \mathrm{vs} \mathrm{Nf}_{\text {lag }}$.

DOI: 10.7554/eLife.01916.017

linking inter-flagellar coupling to the chemotaxis network. We propose that fluctuations in the concentration of CheY-P are at the heart of wild-type E. coli behavior. In our theoretical analysis, the existence of temporal fluctuations was sufficient to explain all of our data. Stochastic simulations with and without CheY-P fluctuations (representing wild-type and $\mathrm{CheY}^{*}$ cells, respectively) reproduced all of the observed differences between our two strains. Figure 4A summarizes how CheY-P fluctuations could lead to 
A

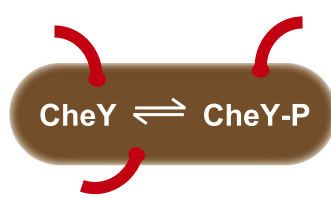

Simulations with CheY-P fluctuations (wild-type)
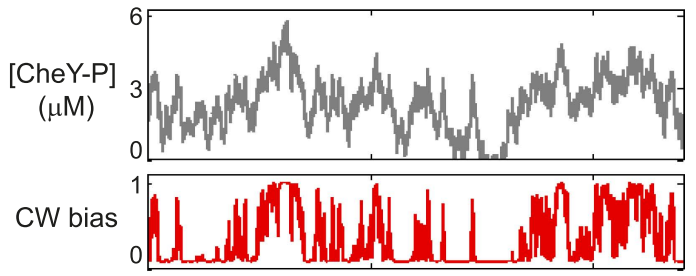

Cell
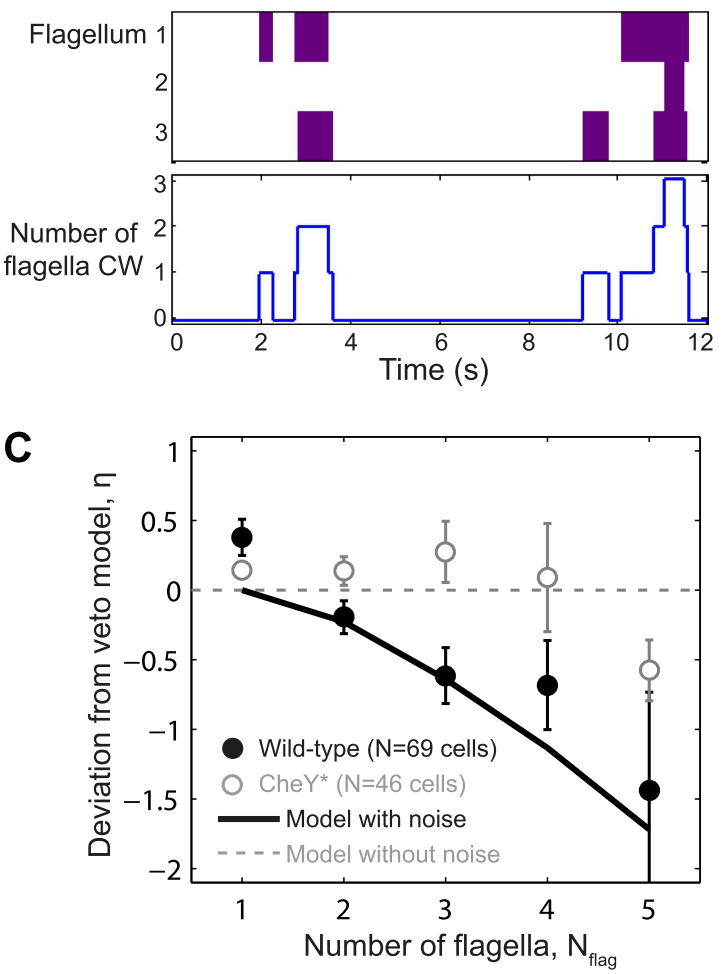

A

Number of flagella, $\mathrm{N}_{\text {flag }}$

C

B

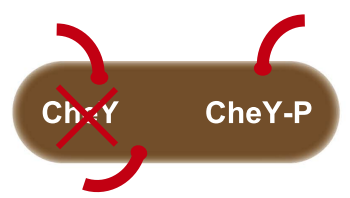

Simulations without fluctuations $\left(\mathrm{CheY}^{*}\right)$
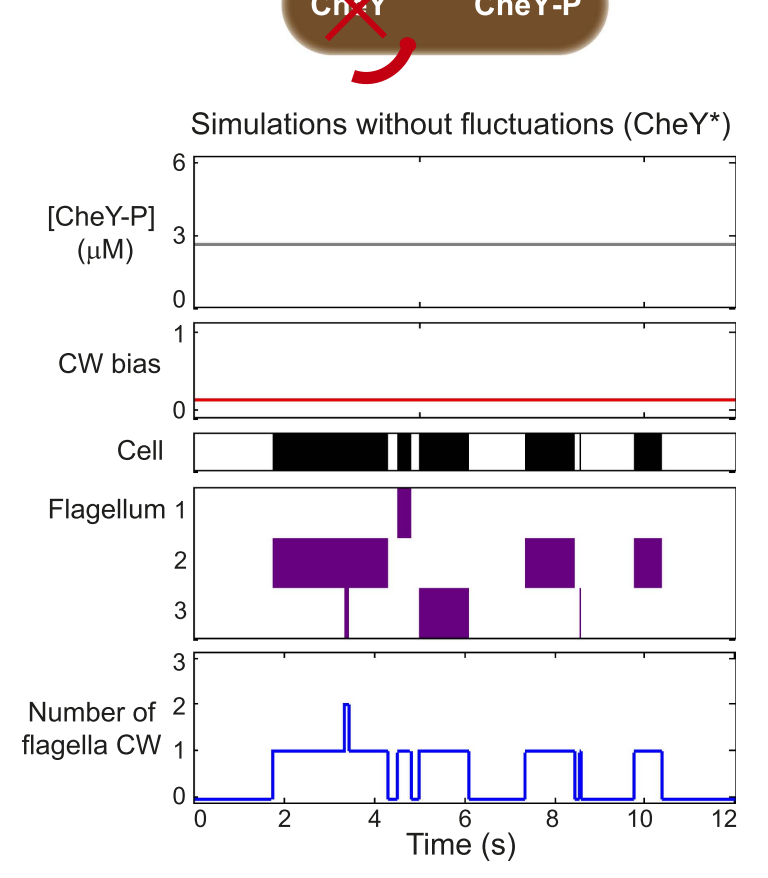

Figure 4. A theoretical model incorporating CheY-P fluctuations reproduces wild-type data. (A) Simulated time trace for a wild-type cell. Representative simulated time trace of CheY-P concentration (gray line, top), CW bias (red), run/tumble state (white/black), CCW/CW flagellar rotational direction (white/ purple) and number of CW flagella (blue line, bottom) for a cell with 3 flagella. CheY-P simulation parameters are described in the text and in Table 3. (B) Same as (A) from a simulated CheY* cell, in which CheY-P concentration (gray line) does not fluctuate. (C) Deviation from veto model. The theoretical model that includes CheY-P concentration fluctuations (black line) reproduces the wild-type data (black circles). A simple veto model with constant CheY-P concentration (gray dashed line) reproduces the CheY^ data (open gray circles). Error bars denote SEM. See 'Materials and methods' for more details.

DOI: 10.7554/eLife.01916.018

The following figure supplements are available for figure 4:

Figure supplement 1. Flagellar waveform transition rates.

DOI: 10.7554/eLife.01916.019

Figure supplement 2. Flagellar waveform transition sequences.

DOI: 10.7554/eLife.01916.020

Figure supplement 3. Flagellar waveform transition sequences.

DOI: 10.7554/eLife.01916.021

Figure supplement 4. Tumble bias vs number of flagella per cell.

DOI: 10.7554/eLife.01916.022 
Table 3. Model parameters

\begin{tabular}{|c|c|c|c|}
\hline Parameter & Description & Value & Source \\
\hline$k_{c c w->c w}$ & Motor switching rate from $\mathrm{CCW} \rightarrow \mathrm{CW}$ & $0.26 \mathrm{~s}^{-1}$ & Our data \\
\hline$k_{\mathrm{cw}->\mathrm{ccw}}$ & Motor switching rate from $\mathrm{CW} \rightarrow \mathrm{CCW}$ & $1.7 \mathrm{~s}^{-1}$ & Our data \\
\hline$C B$ & Average clock-wise bias of wild-type motors & 0.13 & Our data \\
\hline$<Y>$ & Mean concentration of CheY-P & $2.59 \mu \mathrm{M}$ & Fit to our data \\
\hline$\omega$ & Characteristic motor switching time & $0.5 \mathrm{~s}$ & Our data \\
\hline$\lambda^{-1}$ & Transition rate from semi-coiled to curly-1 state & $0.68 \mathrm{~s}^{-1}$ & Our data \\
\hline$x$ & $\begin{array}{l}\text { From the model in Sneddon et al.; number of flagella } \\
\text { that must be normal for a run to occur (while other } \\
\text { flagella are curly-1) (Sneddon et al., 2012) }\end{array}$ & $\begin{array}{l}N_{\text {flag }} \text { (variable in } \\
\text { Figure 1-figure } \\
\text { supplement 5) }\end{array}$ & Our data \\
\hline$\sigma^{2}$ & Variance in [CheY-P] & $1.0 \mu \mathrm{M}^{2}$ & Fit to our data \\
\hline$T$ & Characteristic time-scale of fluctuations in [CheY-P] & $0.2 \mathrm{~s}$ & Fit to our data \\
\hline$K_{d}$ & Midpoint of CW bias vs CheY-P response curve & $3.1 \mu \mathrm{M}$ & (Cluzel et al., 2000) \\
\hline$H$ & Hill coefficient for CW bias vs CheY-P response curve & 10.3 & (Cluzel et al., 2000) \\
\hline$d t$ & Simulation time steps & $0.001 \mathrm{~s}$ & - \\
\hline
\end{tabular}

correlated flagellar switching. A well-known feature of the chemotaxis network is the sigmoidal relation between CW bias and CheY-P concentration (Cluzel et al., 2000; Yuan and Berg, 2013). A consequence of this non-linearity is that the probability of $\mathrm{CW}$ rotation is highly sensitive, and can respond dramatically to fluctuations in CheY-P levels, provided their amplitude is sufficiently large. As shown in Figure 4A, when CheY-P concentration is high, the cell experiences a near-100\% probability of CW rotation and multiple motors switch CW at approximately the same time. In contrast, when CheY-P concentration is low, the probability of any motor rotating CW is essentially zero. This mechanism can explain the elevated number of CW flagella involved in tumbles (Figure 2C) and correlation between flagella states (Figure 2D). By contrast, in simulations where CheY-P level was held constant, flagellar switching was not as correlated and the majority of tumbles involved only a single CW flagellum (Figure 4B).

Despite the success of this model in reproducing our data, we must acknowledge that there is no direct experimental evidence to-date for the CheY-P fluctuations depicted in Figure 4A. Fluctuations in CheY-P have been inferred from experimental observations of CW bias in tethered-bead assays (Korobkova et al., 2004). However, the fluctuations described in that study are different in their time scale and amplitude from what we found required to produce the observed correlations in flagellar rotational direction ('Materials and methods'). Future investigation will be essential to resolving this issue and will likely have to involve direct measurements of CheY-P temporal dynamics in individual cells. Such measurements are challenging, but the development of intra-cellular fluorescence sensors for kinase activity in the network (Sourjik and Berg, 2002) provides a promising approach.

As an alternative mechanism for the inter-flagellar correlations observed by Terasawa et al. (2011) (and in the present work), $\mathrm{Hu}$ and Tu (2013) proposed that hydrodynamic interactions between nearby

Table 4. Flagella waveform transition rates

\begin{tabular}{|c|c|c|c|}
\hline \multirow[b]{2}{*}{ Initial } & \multicolumn{3}{|l|}{ Final } \\
\hline & Normal & Semi-coiled & Curly-1 \\
\hline Normal & & $0.28 \pm 0.03 \mathrm{~s}^{-1}$ & $0.08 \pm 0.01 \mathrm{~s}^{-1}$ \\
\hline Semi-coiled & $1.6 \pm 0.2 \mathrm{~s}^{-1}$ & & $0.54 \pm 0.08 \mathrm{~s}^{-1}$ \\
\hline Curly-1 & $1.8 \pm 0.2 \mathrm{~s}^{-1}$ & $0.04 \pm 0.02 \mathrm{~s}^{-1}$ & \\
\hline
\end{tabular}

Transition rates between different flagellar waveforms: normal (CCW), semi-coiled and curly-1 (both CW). Data from wild-type cells ( $N=52$ cells, 203 tumbles). Values are mean \pm SEM.

DOI: 10.7554/eLife.01916.024 
flagella could also generate correlations in their rotational direction. One consequence of their model is that the flagellar switching rates in a cell with a single flagellum will be different than those in multiflagellated cells. However, this prediction is not borne out by our data. In our experiments, the number of flagella per cell did not have a significant effect on the switching rates between CCW and CW states, nor on the switching rates between different flagellar waveforms (Figure 2-figure supplements 1 and 2). While we cannot rule out the presence of hydrodynamic interactions between flagella, these must satisfy the strict requirement that switching rates remain independent of flagellar number. In light of these constraints, we believe a mechanism in which chemotaxis network fluctuations engender inter-flagella correlations to be more plausible. For cells with $N_{\text {flag }}>4$, we note that both strains appear to deviate from the generalized veto model (Figure 3C). It is possible that hydrodynamic effects must be taken in consideration in cells with many flagella. Hydrodynamic coupling in the $\mathrm{Hu}$ model leads to a lower $N_{\text {eff, }}$ in the direction of the deviation. Alternatively, a mechanism as described by Sneddon et al. (2012), in which cells with many flagella can run while some of its flagella rotate CW in the curly-1 state, could lead to a similar deviation (Figure 1-figure supplement 5). Finally, we must consider the possibility that the apparent deviation is due to systematic experimental error, since it is more difficult to determine visually the state of each flagellum on cells with many flagella.

While a large number of studies have elucidated mathematical relationships between many of the components of the chemotaxis network (Block et al., 1982; Cluzel et al., 2000; Sourjik and Berg, 2002; Shimizu et al., 2010; Yuan et al., 2012), there have been few experimental studies devoted to the relationship between individual flagella and whole-cell swimming (Turner et alo, 2000; Darnton et alo, 2007). As a result, existing models of bacterial chemotaxis have made drastically different assumptions in order to describe the swimming behavior of the whole cell (Bray et al., 2007; Jiang et al., 2010; Vladimirov et al., 2010; Sneddon et al., 2012). To the best of our knowledge, the current study provides the first experimentally-derived mathematical relation between flagellar and whole-cell-swimming states.

We propose that the details of this mapping are crucial for fully understanding bacterial chemotaxis. Results from recent theoretical models suggest that the details of flagellar mechanics can have significant effects on chemotactic drift. Turner et al. observed that, on average, the angular change in swimming direction upon tumbling increases as a function of the number of flagella that leave the bundle (Turner et al., 2000). Vladimirov et al. showed that when this effect is incorporated into a theoretical model of bacterial chemotaxis, the chemotactic drift is nearly doubled (Vladimirov et al., 2010).

Our observations that multi-flagellated wild-type cells tumble significantly less than expected also implies that the cell's swimming behavior (and presumably its chemotactic response) is robust against variations in the number of flagella (Figure 4-figure supplement 4). We hypothesize that this phenomenon may confer evolutionary advantages, in light of the large fluctuations in flagellar numbers within a cell population (Figure 1-figure supplement 1). If cells with many flagella did not behave like cells with fewer flagella, then they would spend the majority of their time tumbling, a behavior that would inhibit chemotaxis. E. coli thus appears to have developed a mechanism to achieve similar tumble biases with a wide range of flagellar number.

\section{Materials and methods}

\section{Microbiology}

\section{Cell preparation}

Experiments were performed using two E. coli strains (Table 1). The strain referred to as 'wild-type' is HCB1660 ([Turner et al., 2010], gift of Howard Berg), $\Delta$ fliC expressing FliC ${ }^{5219 C}$ from a plasmid under the control of arabinose. The mutant protein $\mathrm{FliC}^{\mathrm{S219C}}$ was constructed to be specifically labeled with a maleimide functionalized fluorescent dye (Turner et al., 2010). The strain referred to as ' $\mathrm{CheY}^{\mathrm{Y}^{*}}$ is PM87 (constructed for this study, see below), $\triangle$ fliC $\triangle$ cheBYZ expressing FliC ${ }^{\mathrm{S} 219 \mathrm{C}}$ and CheYD13K from separate plasmids under the control of arabinose and IPTG, respectively. The mutant protein Che ${ }^{D 13 K}$ is constitutively active (Alon et al., 1998), such that the CW bias was determined by the concentration of CheYD13k, decoupled from the chemotaxis network.

For each experiment, cells were picked from a single colony on an agar plate and grown overnight in $1 \mathrm{ml}$ tryptone broth (1\% [wt/vol] Bacto tryptone and 0.8\% [wt/vol] NaCl) (Saini et alo, 2008; Min et alo, 2009) shaking at $265 \mathrm{RPM}$ at $30^{\circ} \mathrm{C}$ with appropriate antibiotics. The overnight culture was diluted 100 -fold into 12-ml tryptone broth and grown, shaking at $265 \mathrm{RPM}$ at $30^{\circ} \mathrm{C}$ for $4.5 \mathrm{hr}$ (to $\mathrm{OD}_{600} \sim 0.5$ ) with appropriate inducers ( $500 \mu \mathrm{M}$ arabinose and $50 \mu \mathrm{M}$ IPTG). To visualize flagella, we used a fluorescence labeling 
protocol developed by Turner et al. (2010). The over-day culture was harvested, washed twice by slow centrifugation (1300 $\times g, 10 \mathrm{~min}$ ) and gently resuspended in $1 \mathrm{ml}$ motility buffer (MB) (Darnton et al., 2007) $\left(10 \mathrm{mM} \mathrm{KPO}_{4}(\mathrm{pH} 7.0), 70 \mathrm{mM} \mathrm{NaCl}\right.$ and $0.1 \mathrm{mM}$ EDTA) and then in $0.5 \mathrm{ml} \mathrm{MB}$. Flagella were specifically labeled using Alexa Fluor $532 \mathrm{C}_{5}$ Maleimide (A-10255; Life Technologies, Carlsbad, California). $1 \mathrm{mg}$ of dry dye was dissolved in $300 \mu \mathrm{l} \mathrm{H}_{2} \mathrm{O}$ by vortexing for $1 \mathrm{~min}$. Aliquots containing $50 \mu \mathrm{l}$ of dissolved dye were stored at $-20^{\circ} \mathrm{C}$. Cells in $500 \mu \mathrm{lMB}$ were gently mixed with $5 \mu \mathrm{l}$ of the dissolved dye and then incubated with slow rotation ( 10 RPM) at room temperature in the dark for $90 \mathrm{~min}$. The labeled culture was washed and gently resuspended in $1 \mathrm{ml} \mathrm{MB}$. Finally, cells were diluted 20-fold into $1 \mathrm{ml}$ trap motility buffer (TMB) $\left(70 \mathrm{mM} \mathrm{NaCl}, 100 \mathrm{mM}\right.$ Tris-Cl, 2\% [wt/vol] glucose, and an oxygen-scavenging system [ $80 \mu \mathrm{g} \mathrm{ml}{ }^{-1}$ glucose oxidase and $13 \mathrm{\mu g} \mathrm{ml}^{-1}$ catalase]) (Min et al., 2009) and injected into the flow cell for trapping. At all stages, resuspension by pipetting was avoided to prevent shearing of the flagella (Turner et al., 2000).

\section{Construction of strains}

Bacterial strains and plasmids used in this study are listed in Table 1. Oligonucleotides used for generating mutations and creating plasmids are listed in Table 2. The generalized transducing phage P1 vir was used in all transductional crosses (Thomason et al., 2007). Chromosomal mutations were introduced using a standard $\lambda$ Red recombination method as described by Datsenko and Wanner (2000). All primers were purchased from Integrated DNA Technologies (Coralville, lowa), all sequencing was performed by ACGT (Wheeling, Illinois).

Strain PM87 (referred to as the $\mathrm{CheY}^{\star}$ strain in the text) was created from RP437 (wild-type for chemotaxis) in the following manner. First, $c h e B Y Z$ was replaced by a chloramphenicol resistance cassette with flanking FRT sites using primers SK140F and SK140R along with pKD3 as a template (Datsenko and Wanner, 2000). This deletion was then moved into a clean RP437 using P1 transduction (Thomason et al., 2007), to create SK109 (cheBYZ::C Cm). The chloramphenicol resistance cassette was removed from SK109 using Flp recombinase expressed from pCP20 (Cherepanov and Wackernagel, 1995) to obtain strain SK110 (cheBYZ::FRT). Next, fliC::Tn5 from strain HCB1660 was moved into strain SK110 using P1 transduction to obtain strain SK112. Finally, strain PM87 was created by transforming strain SK112 with plasmids pPM5 and pMS164 to express FliC ${ }^{\mathrm{S} 219 \mathrm{C}}$ and CheY ${ }^{\mathrm{D} 13 \mathrm{~K}}$, respectively.

Standard molecular cloning techniques were used to construct plasmids (Sambrook and Russell, 2001). Primers PM7F and PM7R were used to $P C R$ amplify fliC $C^{5219 C}$ and the $P_{\text {araBAD }}$ promoter from plasmid

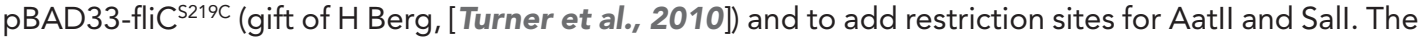
PCR product was then ligated into pZE11 (Lutz and Bujard, 1997) after digesting both with restriction enzymes Aatll and Sall, to obtain plasmid pPM5.

\section{Instrument design}

\section{Optical traps}

Experiments were performed using a dual optical trap instrument incorporating a custom flow cell and stroboscopic, epi-fluorescent imaging. The instrument design is shown in Figure 1-figure supplement 2. The optical trap was constructed following a previously described design (Comstock et alo, 2011). A 5-W, 1064-nm diode-pumped solid-state laser (BL-106C, Spectra-Physics, Santa Clara, California) was used to produce two optical traps via timesharing, by intermittently deflecting the laser with an acousto-optic deflector, AOD (DTD-274HD6, IntraAction, Bellwood, Illinois). The separation between the two traps was controlled by modulating the two deflection angles of the beams emanating from the acousto-optic deflector via the RF signal frequency driving the AOD. The IR beams were tightly focused to generate two optical traps by a 60x, water-immersion (1.2 NA) microscope objective (Nikon, Tokyo, Japan). An identical objective lens collected transmitted light for position detection and bright-field imaging, as described in Min et al. (2009). The flow chamber was positioned between the two objective lenses and was moveable relative to the two traps in all directions by a motorized three-axis translational stage (ESP301; Newport, Irvine, California). Cell motion was detected directly by the optical traps themselves, using back-focal plane interferometry, in which trap light scattered by an object relays the object's position relative to the trap in all three directions (Gittes and Schmidt, 1998). All devices and timing were controlled using custom LabVIEW (National Instruments, Austin, Texas) code (Comstock et al., 2011).

Fluorescence imaging

Epi-fluorescence imaging of trapped cells was achieved by excitation with a 532-nm laser (TECGL-30, World Star Tech, Toronto, Canada) and collecting backwards emitted photons with an EMCCD camera 
(iXon3 860 EMCCD, Andor, Belfast, Ireland). The beam size at the sample plane was approximately 20 microns in diameter. To obtain clear images of the flagella as they rotated at angular frequencies $\sim 100$ $\mathrm{Hz}$, it was necessary to take short exposures (Figure 1-figure supplement 3). To this end, we used stroboscopic illumination similarly to previous experiments (Darnton et alo, 2007). This was achieved by intermittently deflecting the 532-nm laser beam with a second acousto-optic modulator (AOM-802AF1, IntraAction), so as to generate short-duration excitation pulses $(20 \mu \mathrm{s})$. The EMCCD recorded $128 \times$ 128-pixel images $(\sim 10 \times 10 \mu \mathrm{m})$ synchronized with each excitation pulse (see timing in Figure 1-figure supplement 3, images Figure 1-figure supplement 4). Additionally, we pulsed the IR trapping laser out of phase with the fluorescence excitation at a rate of $16 \mathrm{kHz}$, a technique which has been shown to significantly reduce photo-bleaching, with minimal consequence to the trapping (Brau et al., 2006; Comstock et al., 2011) (Figure 1-figure supplement 3). The results were high speed movies showing sharp images of all the flagella on a cell as it runs and tumbles for many seconds (typically $>10$ s), along with synchronous signal traces from the optical traps. Fluorescence movies were saved using Solis software (Andor), and subsequently analyzed manually using Matlab (Mathworks, Natick, Massachusetts). When played back in slow-motion, flagella could be reliably counted by eye during tumbles.

\section{Fluidics}

Experiments were performed in a custom-built microfluidic chamber. See Min et al. (2012) for a detailed description of the microfluidic chamber. Glass coverslips (12-545-M, $24 \times 60-1$, ThermoFisher, Waltham, Massachusetts) were sonicated in acetone for $5 \mathrm{~min}$ and rinsed with deionized water. The flow channel pattern was cut out from Nescofilm (Karlan, Phoenix, Arizona) using a laser engraver (Versa Laser, Scottsdale, Arizona) and placed between two coverslips, one of which had custom-drilled holes (0.05-inch diameter) for inlets and outlets. The Nescofilm flow channel pattern was bonded to coverslips by melting on a hot plate for $4 \mathrm{~min}$. The completed flow chamber was inserted into a custom metal frame where inlet and outlet tubing (ABW00001; Tygon, Saint-Gobain, Paris, France) were screwed on for a tight seal. The two channels of the flow chamber were continuously injected with appropriate buffers using a syringe pump (PHD2000; Harvard Apparatus, Holliston, Massachusetts) at a linear speed of $30 \mu \mathrm{m} / \mathrm{s}$, which is approximately equal to the swimming speed of a healthy cell. The upper channel was injected with TMB (see above), while the bottom channel contained cells in TMB. Cells were trapped in the bottom channel and then moved into the upper channel for observation by displacing the motorized flow chamber relative to the traps.

\section{Electron microscopy}

TEM images were recorded using the JEOL 2100 cryo-Transmission Electron Microscope (TEM) at the Frederick Seitz Materials Research Laboratory Central Facilities at the University of Illinois at UrbanaChampaign, following the protocol of Saini et al. (2010). Briefly, cells were grown as described above, and used without fluorescent labeling. Cells were fixed with glutaraldehyde and then placed on 200 Mesh Carbon Coated Copper grids (Cat. \# 182; Canemco, Lakefield, Canada), which were used as supports for sample loading and imaging. Images were taken at $200 \mathrm{kV}$ with a camera exposure lasting $1 \mathrm{~s}$. Finally, images were contrast adjusted and an image dilation was performed in Matlab to make flagella more visible. The distribution of flagella per cell is shown in Figure 1-figure supplement 1.

\section{Data analysis}

Wavelet analysis for run-tumble detection

Determination of runs and tumbles from the optical trap signal was done as described previously (Min et al., 2009). See example trace in Figure 1C.

Image analysis

Images were contrast adjusted to make flagella easier to see using Matlab. Movies were then manually analyzed by eye to count flagella and to identify flagella waveforms. The state of each flagellum was identified during each $100-\mathrm{ms}$ time window (10 movie frames at 100 f.p.s.). See example cells in Figure $1 \mathrm{C}$ and Figure 1-figure supplement 4.

\section{Theoretical modeling}

\section{The veto model}

The veto model assumes that all flagella must be rotating counter-clockwise (CCW) for the cell to run. Any flagellum can 'veto' the run by rotating clockwise (CW), which causes the cell to tumble (Figure 1A). 
To describe the veto model mathematically, we write the expression for the run bias (the fraction of time that a cell spends running) as a function of the CCW bias (the fraction of time that each flagellum spends rotating $(\mathrm{CW})$. Assuming that the $\mathrm{CCW}$ bias is fixed in time, one obtains:

$$
\begin{gathered}
\text { Run bias }=\frac{\text { Time ALL motors CCW }}{\text { Total time }} \\
\text { Run bias }=(C C W \text { bias })^{N_{\text {flag }}}
\end{gathered}
$$

where $N_{\text {flag }}$ is the number of flagella. We can write the tumble bias (TB) as a function of the run bias, and the $\mathrm{CW}$ bias $(\mathrm{CB})$ as a function of $\mathrm{CCW}$ bias:

$$
\begin{aligned}
& T B=1-\text { RunBias } \\
& C B=1-\text { CCWBias }
\end{aligned}
$$

We then use these relations to solve for the tumble bias as a function of CW bias and the number of flagella:

$$
T B=1-(1-C B)^{N_{\text {flag }}}
$$

\section{Simulating the effect of CheY-P fluctuations}

Stochastic simulations were performed to model the effects of fluctuations in CheY-P concentration over time, using a method similar to Sneddon, et al. (2012). Simulations are illustrated in Figure 4A. CheY-P fluctuations were generated using Equation S6 below (see Table 3 for parameter values), where $Y$ is the instantaneous concentration of CheY-P, $T$ is the characteristic timescale of fluctuations in [CheY-P], $\sigma^{2}$ is the variance in [CheY-P], and $\xi$ are normally distributed random numbers with unit variance. The time resolution of all simulations was one data point per millisecond.

$$
Y(t+d t)=Y(t)-\frac{(Y(t)-(Y))}{\tau} d t+\sigma \sqrt{\frac{2 d t}{\tau}} \xi
$$

The CW bias was calculated from [CheY-P] using the Hill function relationship determined by Cluzel et al. (2000). After generating the CheY-P time trace, the mean CW bias of that trace was checked, to ensure that the bias was within the same range as the data mean (0.13-0.145). The rotational state of each flagellar motor was then determined stochastically, using Equation 1 in Sneddon et al., the CW bias, and characteristic motor switching rate $(\omega)$ to set the CW/CCW transition rates ([Sneddon et al., 2012] see Table 3 for parameter values).

Runs and tumbles were determined using the veto model: whenever any flagellum was rotating $\mathrm{CW}$ the cell tumbled, when all flagella were rotating CCW the cell ran. Finally, the simulated data were analyzed in the exact same manner as the experimental data, to extract the mean number of flagella participating in tumbles (Figure 2C), flagella cross-correlation (Figure 2D), the effective number of

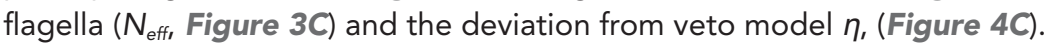

The values of $\sigma$ and $T$, which denote the amplitude and timescale of simulated CheY-P fluctuations, were determined by scanning through a range of values, and minimizing the global, reduced $X^{2}$ (Bevington and Robinson, 2003) from comparisons of simulations to data. The minimum reduced $x^{2}$ value indicates that all of the experimental data is best reproduced by simulations in which $\sigma=1.0 \mu \mathrm{M}^{2}$ and $T=0.2 \mathrm{~s}^{-1}$. The global reduced $\mathrm{x}^{2}$ was calculated by summing the individual reduced $\mathrm{x}^{2}$ values from fits to data in Figures 2C,D and 4C.

Incorporating the effect of curly-1 flagella during runs To test the effect of runs involving curly-1 flagella, we augmented our simulations following Sneddon et al. (2012). For each simulated flagellum, the binary CCW/CW trace was converted into a 3-state (normal, semi-coiled, curly-1) trace, using $\lambda^{-1}=0.68 \mathrm{~s}^{-1}$ as the transition rate from semi-coiled to curly-1 (Table 3). The veto model was then applied, with the caveat that cells ran $18 \%$ of the time when there was a single curly-1 flagellum, provided the other flagella were all CCW (normal) (Figure 1-figure supplement 5). 


\section{Acknowledgements}

We are grateful to Howard Berg, Linda Turner, Vedhavalli Nathan and Philippe Cluzel for advice and for providing reagents. We thank current and former members of the Chemla, Golding, and Rao Laboratories for providing help with experiments.

\section{Additional information}

Funding

\begin{tabular}{|c|c|c|}
\hline Funder & Grant reference number & Author \\
\hline National Science Foundation & PHY-082265 & $\begin{array}{l}\text { Ido Golding, } \\
\text { Yann R Chemla }\end{array}$ \\
\hline Burroughs Wellcome Fund & & Yann R Chemla \\
\hline Alfred P. Sloan Foundation & & Yann R Chemla \\
\hline National Institutes of Health & R01 GM082837 & Ido Golding \\
\hline Welch Foundation & Q-1759 & Ido Golding \\
\hline National Science Foundation & PHY-1147498 & Ido Golding \\
\hline National Institutes of Health & R01 GM054365 & Chris V Rao \\
\hline
\end{tabular}

The funders had no role in study design, data collection and interpretation, or the decision to submit the work for publication.

Author contributions

PJM, Conception and design, Acquisition of data, Analysis and interpretation of data, Drafting or revising the article, Contributed unpublished essential data or reagents; SK, CVR, Conception and design, Drafting or revising the article, Contributed unpublished essential data or reagents; IG, YRC, Conception and design, Analysis and interpretation of data, Drafting or revising the article

\section{References}

Alon U, Camarena L, Surette MG, Aguera y Arcas B, Liu Y, Leibler S, Stock JB. 1998. Response regulator output in bacterial chemotaxis. The EMBO Journal 17:4238-4248. doi: 10.1093/emboj/17.15.4238.

Andrews BW, Yi TM, Iglesias PA. 2006. Optimal noise filtering in the chemotactic response of Escherichia coli. PLOS Computational Biology 2:e154. doi: 10.1371/journal.pcbi.0020154.

Berg HC. 2004. E. coli in motion. New York: Springer.

Berg HC, Brown DA. 1972. Chemotaxis in Escherichia coli analysed by three-dimensional tracking. Nature 239:500-504. doi: 10.1038/239500a0.

Bevington PR, Robinson DK. 2003. Data reduction and error analysis for the physical sciences. 3rd edition. Boston: McGraw-Hill.

Block SM, Segall JE, Berg HC. 1982. Impulse responses in bacterial chemotaxis. Cell 31:215-226. doi: 10.1016/00928674(82)90421-4.

Brau RR, Tarsa PB, Ferrer JM, Lee P, Lang MJ. 2006. Interlaced optical force-fluorescence measurements for single molecule biophysics. Biophysical Journal 91:1069-1077. doi: 10.1529/biophysj.106.082602.

Bray D, Levin MD, Lipkow K. 2007. The chemotactic behavior of computer-based surrogate bacteria. Current Biology 17:12-19. doi: 10.1016/j.cub.2006.11.027.

Cherepanov PP, Wackernagel W. 1995. Gene disruption in Escherichia coli: TcR and KmR cassettes with the option of Flp-catalyzed excision of the antibiotic-resistance determinant. Gene 158:9-14. doi: 10.1016/03781119(95)00193-A.

Cluzel P, Surette M, Leibler S. 2000. An ultrasensitive bacterial motor revealed by monitoring signaling proteins in single cells. Science 287:1652-1655. doi: 10.1126/science.287.5458.1652.

Cohen-Ben-Lulu GN, Francis NR, Shimoni E, Noy D, Davidov Y, Prasad K, Sagi Y, Cecchini G, Johnstone RM, Eisenbach M. 2008. The bacterial flagellar switch complex is getting more complex. The EMBO Journal 27:1134-1144. doi: 10.1038/emboj.2008.48.

Comstock MJ, Ha T, Chemla YR. 2011. Ultrahigh-resolution optical trap with single-fluorophore sensitivity. Nature Methods 8:335-340. doi: 10.1038/nmeth.1574.

Darnton NC, Turner L, Rojevsky S, Berg HC. 2007. On torque and tumbling in swimming Escherichia coli. Journal of Bacteriology 189:1756-1764. doi: 10.1128/Jb.01501-06.

Datsenko KA, Wanner BL. 2000. One-step inactivation of chromosomal genes in Escherichia coli K-12 using PCR products. Proceedings of the National Academy of Sciences of the United States of America 97:6640-6645. doi: 10.1073/pnas.120163297. 
Emonet T, Macal CM, North MJ, Wickersham CE, Cluzel P. 2005. AgentCell: a digital single-cell assay for bacterial chemotaxis. Bioinformatics 21:2714-2721. doi: 10.1093/bioinformatics/bti391.

Flores M, Shimizu TS, ten Wolde PR, Tostevin F. 2012. Signaling noise enhances chemotactic drift of E. coli. Physical Review Letters 109:148101. doi: 10.1103/PhysRevLett.109.148101.

Gittes F, Schmidt CF. 1998. Interference model for back-focal-plane displacement detection in optical tweezers. Optics Letters 23:7-9. doi: 10.1364/OL.23.000007.

Hu B, Tu YH. 2013. Coordinated switching of bacterial flagellar motors: evidence for direct motor-motor coupling? Physical Review Letters 110:158703. doi: 10.1103/Physrevlett.110.158703.

Ishihara A, Segall JE, Block SM, Berg HC. 1983. Coordination of flagella on filamentous cells of Escherichia coli. Journal of Bacteriology 155:228-237.

Jiang LL, Qi OY, Tu YH. 2010. Quantitative odeling of Escherichia coli chemotactic motion in environments varying in space and time. PLOS Computational Biology 6:doi: 10.1371/journal.pcbi.1000735.

Kalinin YV, Jiang L, Tu Y, Wu M. 2009. Logarithmic sensing in Escherichia coli bacterial chemotaxis. Biophysical Journal 96:2439-2448. doi: 10.1016/j.bpj.2008.10.027.

Korobkova E, Emonet T, Vilar JMG, Shimizu TS, Cluzel P. 2004. From molecular noise to behavioural variability in a single bacterium. Nature 428:574-578. doi: 10.1038/Nature02404.

Korobkova EA, Emonet T, Park H, Cluzel P. 2006. Hidden stochastic nature of a single bacterial motor. Physical Review Letters 96:058105. doi: 10.1103/PhysRevLett.96.058105.

Lutz R, Bujard H. 1997. Independent and tight regulation of transcriptional units in Escherichia coli via the LacR/O, the TetR/O and AraC/I1-I2 regulatory elements. Nucleic Acids Research 25:1203-1210. doi: 10.1093/nar/25.6.1203. Macnab RM, Ornston MK. 1977. Normal-to-curly flagellar transitions and their role in bacterial tumbling. Stabilization of an alternative quaternary structure by mechanical force. Journal of Molecular Biology 112:1-30. doi: 10.1016/S0022-2836(77)80153-8.

Matthaus F, Jagodic M, Dobnikar J. 2009. E. coli superdiffusion and chemotaxis-search strategy, precision, and motility. Biophysical Journal 97:946-957. doi: 10.1016/j.bpj.2009.04.065.

Min TL, Mears PJ, Chubiz LM, Rao CV, Golding I, Chemla YR. 2009. High-resolution, long-term characterization of bacterial motility using optical tweezers. Nature Methods 6:831-835. doi: 10.1038/nmeth.1380.

Min TL, Mears PJ, Golding I, Chemla YR. 2012. Chemotactic adaptation kinetics of individual Escherichia coli cells. Proceedings of the National Academy of Sciences of the United States of America 109:9869-9874. doi: 10.1073/ pnas.1120218109.

Parkinson JS, Houts SE. 1982. Isolation and behavior of Escherichia coli deletion mutants lacking chemotaxis functions. Journal of Bacteriology 151:106-113.

Saini S, Brown JD, Aldridge PD, Rao CV. 2008. FliZ is a posttranslational activator of FlhD4C2-dependent flagellar gene expression. Journal of Bacteriology 190:4979-4988. doi: 10.1128/JB.01996-07.

Saini S, Koirala S, Floess E, Mears PJ, Chemla YR, Golding I, Aldridge C, Aldridge PD, Rao CV. 2010. FliZ induces a Kinetic switch in flagellar gene expression. Journal of Bacteriology 192:6477-6481. doi: 10.1128/Jb.00751-10.

Sambrook J, Russell DW. 2001. Molecular cloning: a laboratory manual, 3rd edition. Cold Spring Harbor, NY: Cold Spring Harbor Laboratory Press.

Shimizu TS, Tu Y, Berg HC. 2010. A modular gradient-sensing network for chemotaxis in Escherichia coli revealed by responses to time-varying stimuli. Molecular Systems Biology 6:382. doi: 10.1038/msb.2010.37.

Sneddon MW, Pontius W, Emonet T. 2012. Stochastic coordination of multiple actuators reduces latency and improves chemotactic response in bacteria. Proceedings of the National Academy of Sciences of the United States of America 109:805-810. doi: 10.1073/pnas.1113706109.

Sourjik V, Berg HC. 2002. Receptor sensitivity in bacterial chemotaxis. Proceedings of the National Academy of Sciences of the United States of America 99:123-127. doi: 10.1073/pnas.011589998.

Spiro PA, Parkinson JS, Othmer HG. 1997. A model of excitation and adaptation in bacterial chemotaxis. Proceedings of the National Academy of Sciences of the United States of America 94:7263-7268. doi: 10.1073/pnas.94.14.7263.

Terasawa S, Fukuoka H, Inoue Y, Sagawa T, Takahashi H, Ishijima A. 2011. Coordinated reversal of flagellar motors on a single Escherichia coli cell. Biophysical Journal 100:2193-2200. doi: 10.1016/j.bpj.2011.03.030.

Thomason L, Costantino N, Court D. 2007. E. coli genome manipulation by P1 transduction. Current Protocols in Molecular Biology Chapter 1:Unit 1.17. doi: 10.1002/0471142727.mb0117s79.

Turner L, Ryu WS, Berg HC. 2000. Real-time imaging of fluorescent flagellar filaments. Journal of Bacteriology 182:2793-2801. doi: 10.1128/JB.182.10.2793-2801.2000.

Turner L, Zhang R, Darnton NC, Berg HC. 2010. Visualization of flagella during bacterial Swarming. Journal of Bacteriology 192:3259-3267. doi: 10.1128/JB.00083-10.

Vladimirov N, Lebiedz D, Sourjik V. 2010. Predicted auxiliary navigation mechanism of peritrichously flagellated chemotactic bacteria. PLOS Computational Biology 6:doi: 10.1371/journal.pcbi.1000717.

Vladimirov N, Lovdok L, Lebiedz D, Sourjik V. 2008. Dependence of bacterial chemotaxis on gradient shape and adaptation rate. PLoS Computational Biology 4:e1000242. doi: 10.1371/journal.pcbi.1000242.

Wadhams GH, Armitage JP. 2004. Making sense of it all: bacterial chemotaxis. Nature Reviews Molecular Cell Biology 5:1024-1037. doi: 10.1038/nrm1524.

Yuan J, Berg HC. 2013. Ultrasensitivity of an adaptive bacterial motor. Journal of Molecular Biology 425:1760-1764. doi: 10.1016/j.jmb.2013.02.016.

Yuan J, Branch RW, Hosu BG, Berg HC. 2012. Adaptation at the output of the chemotaxis signalling pathway. Nature 484:233-236. doi: 10.1038/nature10964. 